\title{
Out-of-hospital cardiopulmonary resuscitation in four Serbian university cities: outcome follow-up according to the «Utstein style»
}

SLADJANA ANDJELIC $(\square)$ Institute for Emergency Medical Aid, Belgrade Street Aleksinackih rudara 25/4 11070 New Belgrade, Serbia Phone: +381648243603 E-mail: novizivot@ptt.rs

NATASA DJORDJEVIC Department of Pharmacology and Toxicology, Medical Faculty University of Kragujevac

\author{
SLADJANA ANDJELIC • NATASA DJORDJEVIC
}

\begin{abstract}
Aims. To investigate the return of spontaneous circulation (ROSC), survival to discharge, six-month and one-year survival of patients with out-of-hospital cardiac arrest in four university cities in Serbia.

Methods. A prospective, two-year, multic entre study was designed. Using the Utstein template, we recorded out-of-hospital cardiopulmonary (CPR) and its outcomes, and analyzed the immediate survival (ROSC $>20$ min.), short-term survival (to discharge), long-term survival (one year after discharge), or death following out-of-hospital CPR.

Results. During the study period, 591 patients met the inclusion criteria for enrollment and out-of-hospital CPR. The etiology of arrest was cardiac in $33.8 \%$ of patients. Cardiac arrest was witnessed by the advanced life support (ALS) team in $15.6 \%$ of cases. Asystole was the most frequent initial rhythm at time of arrest $(46.4 \%)$. The highest survival rate $(P<0,001)$ was observed in cases with initial VF and pulseless VT, while patients with asystole and pulseless electrical activity (PEA) had the least chance of survival. Within the whole group of patients, ROSC was detected with a frequency of $69.7 \%$. The frequency of patients who died in the field or during admission to hospital was $58.9 \%$, while $28.6 \%$ of admitted patients died before discharge. Thirteen percent of patients survived until discharge, and the overall six-month and one-year survival rates were $11.3 \%$ and $10 \%$, respectively. No statistical difference in survival rates among the cities was found

Conclusion. The Utstein template should be implemented in the form of an official protocol for out-of-hospital CPR follow-up in all emergency medical services in Serbia.
\end{abstract}

Key words: out-of-hospital CPR, outcome, follow-up, Utstein style, Serbia

\section{Introduction}

Sudden cardiac arrest is considered a leading cause of death in the world, accounting for 700,000 deaths annually in Europe alone. It occurs most often in the out-of-hospital environment, with an incidence of 38 for every 100,000 inhabitants. (1) Yet, patients experiencing sudden cardiac arrest could be saved if cardiopulmonary resuscitation (CPR) is started early.

The actual efficacy of out-of-hospital CPR in Serbia, as well as in many other countries, is still unknown. The results of previous studies investigating this phenomenon vary, as they have been conducted in different populations and with different types of emergency care, or investigated different types of cardiac arrest or different protocols. To overcome these problems, the so-called Utstein style has been created, representing the collection of uniform terms and definitions used for analyzing and reporting on out-ofhospital CPR. (2) Data on each of the out-of-hospital cardiac arrest (OHCA) outcomes can be used for standard reporting of $\mathrm{OHCA}$, or included in the Utstein style template for recording cardiac arrest data. The standard report consists of CPR variables that predict out-of-hospital CPR outcome. The Utstein guidelines represent the original method for emergency medi- 
cal service (EMS) systems evaluation. The applicability of the Utstein style methodology enabled a number of communities to identify flaws in their EMS system as well as to modify and optimize the treatment of critical outof-hospital patients.

The aim of our study was to investigate the return of spontaneous circulation (ROSC), survival to discharge, six-month and one-year survival of patients with OHCA in four university cities in Serbia, according to the Utstein style.

\section{Materials and methods}

Study design

The study was conducted prospectively during a two-year period, from 1 January 2007 to 31 December 2008. This multi-centre investigation involved four Serbian university cities with similar organization of the EMS system: Belgrade (BG), Novi Sad (NS), Nis (NI) and Kragujevac (KG).

Belgrade, Nis, Novi Sad and Kragujevac consist of urban, suburban and semirural communities. Belgrade is the capital of Serbia with an area of $3,222.7 \mathrm{~km}^{2}$ and a population of $1,576,124$. Novi Sad is the second largest city in Serbia; it covers an area of $129.4 \mathrm{~km}^{2}$ and has 298.139 residents. Nis covers an area of $596.7 \mathrm{~km}^{2}$ with 250,518 residents. Kragujevac covers an area of 835.0 $\mathrm{km}^{2}$, and has 180,252 inhabitants. Outof-hospital CPR and its outcomes were recorded.

Using the Utstein template, the distribution of patients and the events during out-of-hospital CPR and follow-up were analyzed in a whole study sample, as well as for each city independently: BG, $\mathrm{NS}, \mathrm{NI}$, and KG. The data are presented in figure 1. During the study, 591 patients met the inclusion criteria for enrollment and out-of-hospital CPR. In the study sample, $67 \%$ were men, and the median age was $62.3 \pm 15.3$ years (range 19 - 90 years).

The prehospital advanced life support (ALS) team provided ALS according to the ERC CPR Guidelines for 2005.

(3) Telephone follow-up was used to determine 1-year survival rates.

The study was approved by the Ethics
Committee of the Serbian Medical Society in Belgrade, Serbia.

The study sample consisted of patients older than 18 years of age who had experienced a sudden OHCA, regardless of the etiology or initial electrocardiogram. ALS resuscitation was performed in the following events: when the attending physician diagnosed cardiac arrest upon arrival, or when a hemodynamically unstable or critical patient experienced a cardiac arrest in the presence of the ALS team. Exclusion criteria were in accordance with the Utstein style JRCALC guidelines 1996. (4)

Using the Utstein template, we analyzed three different categories of variables:

1. Patient variables,

2. Cardiac arrest variables, such as etiology, witnesses, initial rhythm (pulseless ventricular fibrillation (VF) / ventricular tachycardia (VT) or non-VFNT), and

3. Outcome variables, such as immediate survival (ROSC >20 min.), shortterm survival (to hospital discharge HD), long-term survival (one year after discharge), or death after the out-ofhospital CPR.

Data were collected by the ALS physician in the prehospital setting using detailed protocols for data collection, patient hospital records, and telephone interviews with the patient, the patient's relatives, and the patient's general practitioner. A presumed etiology of cardiac arrest was determined using all available information, including death certificates and autopsy reports. Data analyses and telephone interviews were performed between 1 January 2007 and 31 December 2008. The primary end-points of the study were death, survival to discharge, and six-month and one-year survival after discharge from the hospital.

Coded data were used for creating the database on a personal computer. For statistical analysis SPSS software (version 12.01, SPSS Inc., Chicago, IL, USA) (5) was used.

Comparison of the individual risk factors was conducted using the $\chi^{2}$-test (Fisher's exact test) or Student t-test (for parametric data). For all statistical procedures, $\mathrm{P}<0.05$ was considered significant. The results represent the descriptive presentation of the Utstein template data, as well as one-way ANOVA of the individual risk factors.

\section{Results}

The etiology of arrest was cardiac in $33.8 \%$ of the whole group of patients (table 1), and 33.7\%, 41.4\%, 33.8\% and $25.6 \%$ of the patients in BG, NS, NI and $K G$, respectively. Within the whole study sample, cardiac arrest was witnessed by the ALS team in $15.6 \%$ of cases. The initial rhythm of arrest was classified as VF in $24.7 \%$, pulseless VT in $5.2 \%$, pulseless electrical activity (PEA) in $23.6 \%$, or asystole in $46.4 \%$. Asystole was the most frequent initial rhythm at time of arrest, with frequencies slightly different among the cities: BG $-42.4 \%$, NS $46.5 \%, \mathrm{NI}-45.7 \%$ and $\mathrm{KG}-59.3 \%$. Considering the initial rhythm at the time of arrest, significant differences in all survival rates (survival to discharge, six-month and one-year survival after discharge) were detected $(P<0,001)$. The highest survival rate was observed in cases of VF and pulseless VT, while patients with asystole and PEA had the least chance of survival (table 2). Within the whole group of patients, ROSC was detected with a frequency of $69.7 \%$. The frequency of patients who died in the field or during admission to hospital was $58.9 \%$, with significant differences among the cities: BG - 48.6\%, NS $54.5 \%, \mathrm{NI}-71.5 \%$ and $\mathrm{KG}-72.1 \%$.

The overall percentage of patients admitted to hospital was $41.1 \%$. During the first 24 hours after admission $22.3 \%$ of patients died, while another $28.6 \%$ of admitted patients died before discharge. In total, $12.5 \%$ of patients survived till discharge. The overall six-month survival rate was $11.3 \%$. One-year survival rate was $9.98 \%$. Figure 1 shows the data in detail. No statistical difference in survival rates among the cities was found (ROSC: $P=0.052$, HD: $P=0.365$, $12 \mathrm{~m}: \mathrm{P}=0.469)$.

\section{Discussion}

To the best of our knowledge, this is 


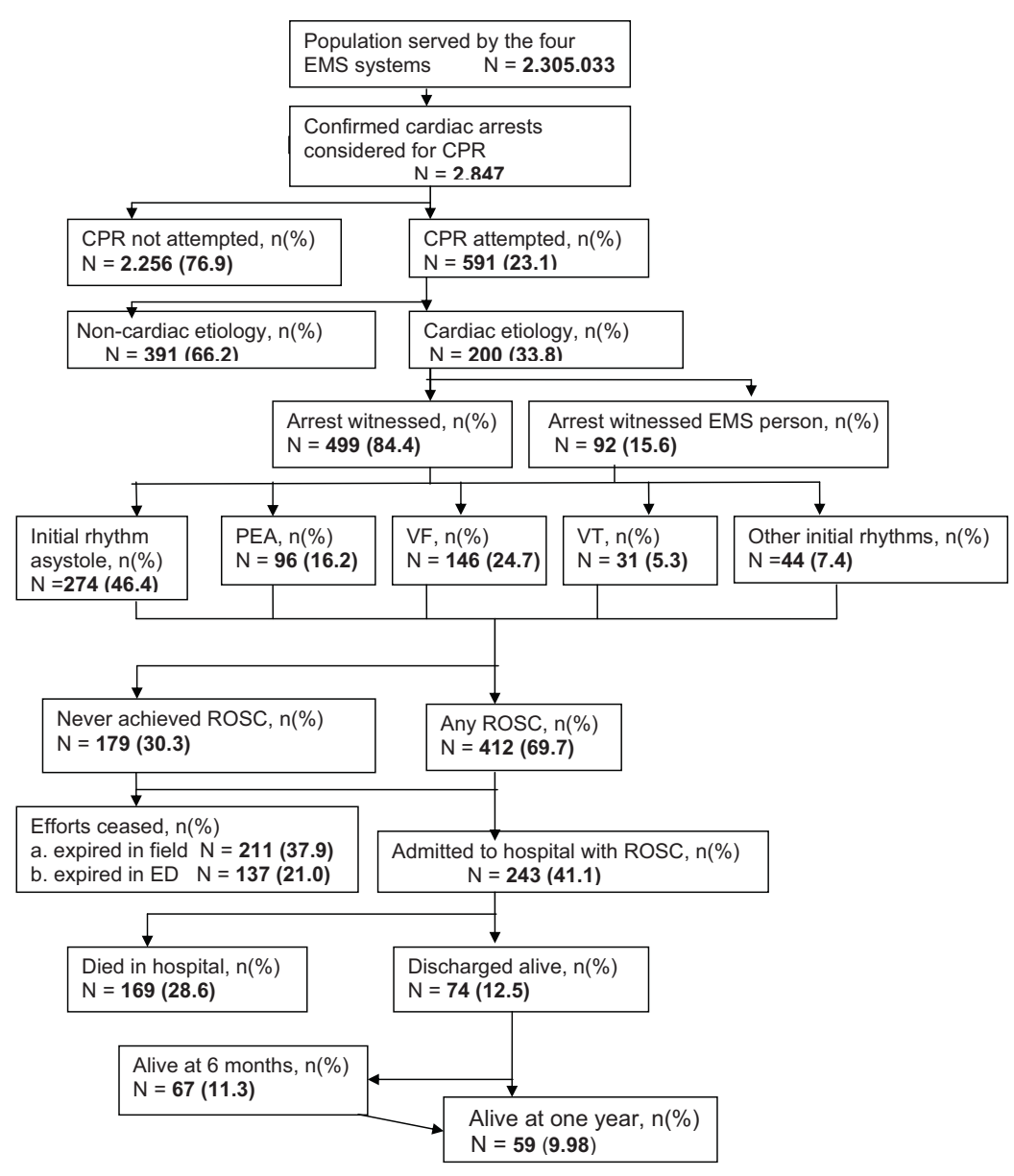

Figure 1. The Utstein template data, total sample.

Legend: CPR - cardiopulmonary resuscitation; EMS - emergency medical services; $\mathrm{N}$ - number; PEA - Pulseless electrical activity; ROSC - return of spontaneous circulation; VF - ventricular fibrillation; VT - ventricular tachycardia.

the first study reporting on out-of-hospital CPR and its outcome in Serbia according to the Utstein style. The incidence of out-of-hospital CPR in Europe (6) ranges between 49.5 and 66 per 100,000 inhabitants annually, which significantly differs from our results of 23.1 per 100,000. Deficiency of the National OHCA Registry is the reason why the incidence of OHCA in our study is much lower than that of western countries.

Immediate, short-term and long-term survival after OHCA varies with the organization of the health system. Our practitioners report ROSC in $69.7 \%$ of patients in the field, with $41.1 \%$ of cases with spontaneous circulation withheld other investigators, e.g. Schoenenberger (23\%), (11) Lewis (21.7\%), (12) and Grmec (21\%). (13) The one-year survival rate detected in each of the cities we investigated corresponds well with European data accumulated in the last 24 years and presented on Medline (table 4). (14) In spite of the financial problems and the lack of facilities that the EMS in Serbia is facing, we consider its efficacy satisfying. The studies from Seattle and Miami reported a one-year survival rate of up to $30 \%$. (15)

Several key variables for a better $\mathrm{OHCA}$ outcome, i.e. cardiac etiology of arrest, pulseless VT as the initial rhythm of arrest, witnessed cardiac arrest and male sex, have been detected. In the present study, cardiac etiology was confirmed in $33.8 \%$ of patients, while a similar study from Seattle reported acute coronary syndrome as the cause of cardiac arrest in $78 \%$ of cases. In comparison to other causes of OHCA, a cardiac etiology was linked to a statistically different survival rate (table 1).

In contrast to the MONICA-project presenting VFNT as the most frequent initial rhythm in OHCA, we found asystole in $46,4 \%$ of cases, VF in $24.7 \%$, PEA in $23.6 \%$, and pulseless VT in $5.2 \%$ of our study subjects. The late arrival of the medical emergency team to the field might be an explanation for the high frequency of asystole and PEA. A previous study from a Japanese group, (16) designed as a comparison between national and international data, showed an $11.2 \%$ incidence of out-of-hospital VF in Okayama (Japan), which is considerably lower than that in western European cities (Oakland 50.9\%, Helsinki 49.8\%, Bon 25.6\%, Glamorgan $21.8 \%)$. We found a statistically significant difference in all investigated survival rates depending on the initial rhythm (ROSC: $P<0.001, H A: P<0.001,12 m$ : $P<0.001$ ), with the highest percentage of survivals after pulseless VT, and the lowest after asystole and PEA (table 2). In two thirds of cases there was a witness to the OHCA, and the survival of those patients depended on early CPR conducted by laypersons. The bystander layperson in our study generally 
Table 1. Etiology of out-of-hospital cardiac arrest (OHCA) and survival.

\begin{tabular}{|l|l|l|l|l|}
\hline Immediate cause & $\mathbf{n}(\%)$ & ROSC $\mathbf{~ ( \% ) ~}$ & HD n (\%) & 1-year $\mathbf{~ ( \% ) ~}$ \\
\hline Cardiac etiology & $200-33.8$ & $65-32.5$ & $40-20.0$ & $34-17.0$ \\
\hline Non-cardiac etiology & $140-23.7$ & $42-30.0$ & $31-22.1$ & $24-17.1$ \\
\hline Unknown & $251-42.5$ & $15-6.0$ & $3-12.0$ & $1-0.4$ \\
\hline$\Sigma$ & $591-100$ & $122-20.6$ & $74-12.5$ & $59-10.0$ \\
\hline $\mathbf{P}$ & & $<0,001$ & $<0,001$ & $<0,001$ \\
\hline
\end{tabular}

HD- hospital discharged alive, n- number of patients, \%- percent, ROSC- admitted to hospital with return of spontaneous circulation, 1-year- alive at one year after OHCA.

Table 2. Arrest rhythms and survival.

\begin{tabular}{|l|l|l|l|l|}
\hline Arrest rhythms & $\mathbf{n ~ ( \% )}$ & ROSC $\mathbf{n}(\%)$ & HD $\mathbf{n}(\%)$ & 1-year $\mathbf{n}(\%)$ \\
\hline VF & $146-24.7$ & $52-35.6$ & $33-22.6$ & $29-19.9$ \\
\hline pulseless VT & $31-5.2$ & $17-54.8$ & $12-38.7$ & $11-35.5$ \\
\hline PEA & $140-23.6$ & $40-28.5$ & $24-17.1$ & $16-11.4$ \\
\hline Asystole & $274-46.4$ & $13-4.7$ & $5-1.8$ & $3-1.1$ \\
\hline$\Sigma$ & $591-100$ & $22-20.6$ & $74-12.5$ & $74-12.5$ \\
\hline $\mathbf{P}$ & & $<0,001$ & $<0,001$ & $<0,001$ \\
\hline
\end{tabular}

HD- hospital discharged alive, n- number of patients, \%- percent, VF - ventricular fibrillation, VT - ventricular tachycardia, ROSCadmitted to hospital with return of spontaneous circulation, 1-year - alive at one year after OHCA.

Table 3. Percentage of immediate and short-term survival after OHCA in different studies. (8-14)

\begin{tabular}{|l|l|l|}
\hline Study & ROSC (\%) & HD (\%) \\
\hline Lazon AJ (8) & 38 & 15 \\
\hline Shih CL (9) & 39 & 17 \\
\hline Lewis et al. (12) & 34 & 21.7 \\
\hline Peatfield et al. (10) & 29 & 27 \\
\hline Schoenenberger (11) & 28 & 23 \\
\hline Summary data from 37 EMS in Europe (14) & 20.7 & 10.7 \\
\hline Grmec S et al. (13) & 61 & 21 \\
\hline Our study & 41.1 & 12.5 \\
\hline
\end{tabular}

HD - hospital discharge, ROSC - Return of Spontaneous Circulation. 
Table 4. Survival rate after OHCA in our and other European cities. (14)

\begin{tabular}{|l|l|}
\hline City & $\begin{array}{l}\text { One year } \\
\text { survival } \\
\text { rate (\%) }\end{array}$ \\
\hline Stavanger & 23 \\
\hline Mainz & 19 \\
\hline Göttingen & 18 \\
\hline Helsinki & 17 \\
\hline Reykjavik & 16 \\
\hline Bonn & 15 \\
\hline Copenhagen & 13 \\
\hline Gothenburg & 12 \\
\hline Madrid & 12 \\
\hline Novi Sad & $\mathbf{1 2}$ \\
\hline Antwerp & 11 \\
\hline Amsterdam & 11 \\
\hline Beograd & $\mathbf{1 1}$ \\
\hline Berlin & 9 \\
\hline Nis & $\mathbf{9}$ \\
\hline Oslo & 8 \\
\hline Bruges & 8 \\
\hline Udine & 8 \\
\hline Kragujevac & $\mathbf{6}$ \\
\hline
\end{tabular}

included family members, neighbors or colleagues at work. The proportion of witnessed cases varied between $15.6 \%$ (EMS person) and $84.4 \%$ (layperson). Bystander CPR by a layperson was performed in only $16.2 \%$ of cases. The reason is insufficiently trained bystanders in Basic Life Support in Serbia. Comparison of survival rates in witnessed and non-witnessed OHCA showed no signi- ficant (ns) difference (ROSC: $P=0.213$, HD: $P=0.601,12 \mathrm{~m}: P=0.957)$. Previous studies reported similar (ns) results in Houston, Chicago, Los Angeles, and New York. (17)

In the present study, the male sex correlated with a higher survival rate, having a frequency of survival after out-of-hospital cardiac arrest of $75 \%$. This is in accordance with a number of previous reports, except for one study from Sweden reporting female sex as a predictor of a higher rate of survival. (18) The studies on the influence of age on the survival rate after OHCA have yielded controversial results. Several papers, $(19,20)$ yet not all of them, reported old age as a bad prognostic sign. The age range of our patients was 20 - 96 years, with an average of $62.3 \pm 15.3$. We did not find age to be an important factor for survival. This is in agreement with several previous studies, (21-23) but in contrast to the BRESUS study, (24) which reported age to be of importance for OHCA outcome.

Our study sample consisted of patients experiencing OHCA regardless of the cause, which makes our study unbiased and objective and the sample itself representative in regard to both patient characteristics and sample size. The smallest study presented so far had 26 cases of OHCA, (23) while the largest study, which lasted for seven years, involved 21,175 OHCA cases from Scotland.

To avoid the discrepancy between urban and rural areas regarding survival rates after $\mathrm{OHCA}$, we conducted our study in four university cities in Serbia. These cities are different in terms of the number of inhabitants, yet similar in terms of the organization of the emergency medical service system. The difference in the survival rates among the cities was non-significant, which confirms the validity of the choice. The highest immediate survival rate among the cities was seen in Belgrade (51.4\%, table 5). This might be due to the highest number of ambulance crews, a modern dispatcher centre, continual medical education, as well as due to the betterequipped ambulance vehicles available in Belgrade. However, a number of patients died during transport (impossibility to continue all established monitoring and supportive therapy) or admission to the hospital emergency department (re-arrest, recurrent VF, hemodynamic instability, etc.). Serbian CPR protocols are not associated with the prehospital use of induced hypothermia for cardiac arrest victims by emergency physicians.

Short-term survival rates were found to be almost identical between Belgrade (14.5\%) and Novi Sad (14.1\%). Discharged patients spent 22.1 days in hospital on average, which is twice as long as reported in Scotland (10 days). Follow-up revealed the highest survival rates in Novi Sad, with $13.1 \%$ and $12.1 \%$ of patients alive six months and one year after OHCA, respectively. We assume that post-resuscitation therapeutic hypothermia, available at the Cardiology Clinics in Sremska Kameni-

Table 5. Survival after OHCA in four cities in Serbia.

\begin{tabular}{|l|l|l|l|l|}
\hline City & ROSC (\%) & HD (\%) & $\begin{array}{l}\text { Alive at 6 months } \\
(\%)\end{array}$ & Alive at 1 year (\%) \\
\hline Belgrade & 51.4 & 14.5 & 12.9 & 11.0 \\
\hline Novi Sad & 45.5 & 14.1 & 13.1 & 12.1 \\
\hline Nis & 28.5 & 10.6 & 9.9 & 9.3 \\
\hline Kragujevac & 27.9 & 8.1 & 7.0 & 5.8 \\
\hline$\sum$ & 41.1 & 12.5 & 11.3 & 10.0 \\
\hline$P(n s)$ & 0.052 & 0.365 & 0.422 & 0.469 \\
\hline
\end{tabular}

HD - hospital discharge, ns - non significant, OHCA - out of hospital cardiac arrest, ROSC - return of spontaneous circulation. 
ca near Novi Sad, is responsible for the described difference.

Yet, there are other reports that differ from our study. For example, the study from Victoria showed that out of-hospital CPR was more successful in the capital in comparison to rural communities. (25) On the other hand, the PHASE study reported lowest survival rates in the biggest cities comparing to more rural areas. (26)

In the present study, a lethal outcome following OHCA was observed on the field in $37.9 \%$ of patients, on admission to hospital in $2.0 \%$, during the hospital stay in $28.6 \%$, and within the one-year period after discharge from hospital in
$2.5 \%$. In the study by Herlitz, $65.5 \%$ of all deaths were in the field, which makes this location a bad prognostic sign for survival. (27)

The cause of death was confirmed by autopsy in 86 patients, and the results correspond well to previously published data. (28) The most frequent cause of death, found in 46 patients (53.5\%), was ischemic heart disease, while trauma (14.0\%) and other causes (32.5\%) were less frequent.

\section{Conclusion}

The outcomes of out-of-hospital CPR and its survival rates, as investigated in this two-year study, are not significantly different among four Serbian cities: Belgrade, Novi Sad, Nis and Kragujevac (ROSC; HD; 12m). The factors influencing the outcome of out-of-hospital CPR are witnessed cardiac arrest, cardiac etiology of arrest, VT/VF as the initial rhythm of arrest and male sex. Our study shows that the Utstein template might be implemented in the form of an official protocol for out-of-hospital CPR follow-up in the entire EMS in Serbia. At the same time, the establishment of a National Registry of OHCA would help detect possible deficiencies of out-ofhospital CPR performance throughout our country and enable reorganization and quality improvement.

\section{REFERENCES}

1. Straus S, Bleumink G, Dieleman J, Lei van der J, Stricker B, Sturkenboom M. The incidence of sudden cardiac death in the general population. J Clin Epidemiol 2004;57(1):98-102.

2. Cummins RO, Chamberlain D, Hazinski MF, Nadkarni V, Kloeck W, Kramer E, et al. Recommended guidelines for reviewing, reporting, and conducting research on in-hospital resuscitation: the in-hospital 'Utstein style.' American Heart Association. Circulation 1997;95:2213-39.

3. Nolan J. European Resuscitaion Council Guidelines for Resuscitation 2005. Section 1. Introduction. Resuscitation 2005;67S1:53-6.

4. Baskett P, Fisher J, Marsden A. Recognition of death by ambulance personnel. Joint Royal Colleges Ambulance Liaison Committee Newsletter. 1996:1.

5. Hilbe JM. A Review of SPSS 12.01. Am Stat 2004;58:168-71.

6. Straus S, Bleumink G, Dieleman J, Lei van der J, Stricker B, Sturkenboom M. The incidence of sudden cardiac death in the general population. J Clin Epidemiol 2004;57(1):98-102.

7. Kass Le, Eitel DR, Sabulsky NK, Ogden CS, Hess DR, Peters KL. One-year survival after prehospital cardiac arrest: the Utstein style applied to a rural-suburban system. Am J Emerg Med 1994;12(1):17-20

8. Lazon AJ, Gabrielli A, Goldfeder BW, Hevia A, Idris AH. Utstein style analysis of rural out-of-hospital cardiac arrest [OOHCA]: total cardiopulmonary resuscitation (CPR) time inversely correlates with hospital discharge rate. Resuscitation 2003; 56(1):59-66

9. Shih CL, Lu TC, Jerng JS, Lin CC, Liu YP, Chen WJ, et al. A Web-base Registry System on Out-of'Hospital Resuscitation Using Utstein style in Taiwan. Resuscitation 2007;72(3):394-403

10. Johnson AL, Tanser PH, Ulan RA, Wood TE. Results of cardiac resuscitation in 552 patients. Am J Cardiol 1967;20:831-5.

11. Schoenenberger RA, von Planta M, von Planta I. Survival after failed out of hospital resuscitation. Arch Intern Med 1994;154:2433-5.

12. Lewis LM, Ruoff B, Rush C, Stothert JC. Is emergency department resuscitation of out of hospital cardiac arrests victims who arrive pulseless worthwhile? Amer J Emerg Med 1990;8:118-20

13. Grmec S, Krizmaric M, Mally S, Kozelj A, Spindler M, Lesnik B. Utstein style analysis of out-of-hospital cardiac arrest - where are bystander CPR-s? Resuscitation 2007;72:404-14

14. Atwood C, Eisenberg MS, Herlitz J, Rea TD. Incidence of EMS-treated out-of-hospital cardiac arrest in Europe. Resuscitation. 2005;67(1):75-80.

15. Liberthson RR, Nagel EL, Hirschman JC, et al. Prehospital ventricular fibrillation: prognosis and follow-up course. N Engl J Med. 1974;291:317-21.

16. Hayashi H. i Ujike Y.Out-of-hospital cardiac arrest in Okayama city (Japan): Outcome reportaccording to the Utstein style. Acta Med. Okayama. 2005;59(2):49-54.

17. Fredriksson M. Variation in outcome in studies of out-of-hospital cardiac arrest: a review of studies conforming to the Utstein guidelines. Am J Emerg Med. 2003; 21(4):276-81.

18. Herlitz J, Engdahl J, Svensson L, Young M, Änquist K, Holmberg S. Is female sex associated with increased survival after out-of-hospital cardiac arrest? Resuscitation 2004;60(2):197-203. 
19. Murphy DJ, Murray AM, Robinson BE, Campion EW. Outcomes of cardiopulmonary resuscitation in the elderly. Ann Intern Med 1989;111:199-205.

20. Rogove HJ, Safar P, Sutton-Tyrell K, Abramson NS. Old age does not negate good cerebral outcome after cardiopulmonary resuscitation: Analyses from the brain resuscitation clinical trials. Crit Care Med 1995;23:18-25.

21. Kellermann AL, Hackman BB, Somes G. Predicting the outcome of unsuccessful pre-hospital advanced cardiac life support. JAMA 1993;270:1433-6.

22. Lewis LM, Ruoff B, Rush C, Stothert JC. Is emergency department resuscitation of out-of-hospital cardiac arrest victims who arrive pulseless worth-while? Am J Emerg Med 1990;8:118-20.

23. Juchems R, Wahlig G, Frese W. Influence of age on survival rate of out-of-hospital and in-hospital resuscitation. Resuscitation 1993;26:23-9.

24. Tunstall-Pedoe H, Bailey L, Chamberlain DA, Marsden AK, Ward ME, Zideman DA. Survey of 3765 cardiopulmonary resuscitations in British hospitals (the BRESUS study): Methods and overall results. BMJ 1992;304:1347-51.

25. Jennings $P$, Cameron P, Walker T, Bernard S, Smith K. Out-of-hospital cardiac arrest in Victoria: rural and urban outcomes. MJA 2006;185(3):135-9.

26. Lombardi G, Gallagher J, Gennis P. Outcome out-of-hospital cardiac arrest in New York City: the Pre-Hospital Arrest Survival Evaluation (PHASE) Study. JAMA 1994;271:678-83.

27. Herlitz J, Eek M, Holmberg M, Engdahl J, Holmerg S. Characteristics and outcome among patients having out of hospital cardiac arrest at home compared with elsewhere. Heart 2002;88(6):579-82.

28. Vanbrabant $\mathrm{P}$, Dhondt E, Billen $\mathrm{P}$, Sabbe M. Etiology of unsuccessful prehospital witnessed cardiac arrest of unclear origin. Eur J Emerg Med 2006;13(3):144-7. 